Centrum voor Wiskunde en Informatica

REPORT RAPPORT

CW

A limit theorem for solutions of inequalities

I.S. Molchanov

Department of Operations Reasearch, Statistics, and System Theory

BS-R9515 1995 
Report BS-R9515

ISSN 0924-0659

CWI

P.O. Box 94079

1090 GB Amsterdam

The Netherlands

$\mathrm{CWI}$ is the National Research Institute for Mathematics and Computer Science. CWI is part of the Stichting Mathematisch Centrum (SMC), the Dutch foundation for promotion of mathematics and computer science and their applications.

SMC is sponsored by the Netherlands Organization for Scientific Research (NWO). CWI is a member of ERCIM, the European Research Consortium for Informatics and Mathematics.

Copyright @ Stichting Mathematisch Centrum P.O. Box 94079, 1090 GB Amsterdam (NL) Kruislaan 413, 1098 SJ Amsterdam (NL) Telephone +3120 5929333 Telefax +3120 5924199 


\title{
A Limit Theorem for Solutions of Inequalities
}

\author{
Ilya S. Molchanov \\ $C W I$ \\ P.O. Box 94079, 1090 GB Amsterdam, The Netherlands \\ ilia@cwi.nl
}

\begin{abstract}
Let $H(p)$ be the set $\{x \in X: h(x) \leq p\}$, where $h$ is a real-valued lower semicontinuous function on a locally compact second countable metric space $X$. A limit theorem is proved for the empirical counterpart of $H(p)$ obtained by replacing of $h$ with its estimator.

AMS Subject Classification (1991): 52A22, 60D05, 60F05, $62 \mathrm{G} 99$.

Keywords 83 Phrases: Aumann expectation, polar set, random set, Hausdorff metric, weak convergence

Notes: The author was supported by the Netherlands Organization for Scientific Research (NWO). This paper has been submitted for publication.
\end{abstract}

\section{INTRODUCTION}

Consider a certain lower semicontinuous real-valued function $h$ defined on a locally compact second countable metric space $(X, \rho)$. Then the set

$$
H(p)=\{x \in X: h(x) \leq p\}
$$

is closed. The aim of this paper is to prove a limit theorem for the estimator $H_{n}(p)$ of the set $H(p)$ obtained by replacing $h(x)$ in (1.1) with its estimator $h_{n}(x)$ :

$$
H_{n}(x)=\left\{x \in X: h_{n}(x) \leq p\right\} .
$$

A simple problem of this kind originates in classical statistics.

ExAmple 1.1. Suppose that $h(x)=F(x), x \in \mathbf{R}$, is the distribution function of a random variable $\nu$. Then $H(p)=\left(-\infty, x_{p}\right]$, where $x_{p}$ is the $p$-quantile of $\nu$, and $H_{n}(p)$ is related to the corresponding empirical quantile if $h_{n}$ is the empirical distribution function. A generalization for quantiles of random vectors and random closed sets was considered by Eddy [2] and Molchanov [7]. 
If $h$ is a density, then the level set $H(p)$ appears in cluster analysis, see Hartigan [3]. An estimator of $H(p)$ based on minimization of the so-called excess mass was considered in $[4,9]$. Similar problems appear also in the estimation of the support of a density, see [5].

Further we shall not discuss the nature of the estimator $h_{n}$. We only suppose that the estimator $h_{n}$ is strongly consistent in the uniform metric and

$$
\zeta_{n}=a_{n}\left(h_{n}-h\right)
$$

admits a weak limit $\zeta$ as $n \rightarrow \infty$, i.e. each continuous in the uniform metric functional of $\zeta_{n}$ converges in distribution to its value on $\zeta$. Here $a_{n} \rightarrow \infty$ as $n \rightarrow \infty$ is a sequence of norming constants.

Suppose also that each function $h_{n}$ is almost surely lower semicontinuous. Then $H_{n}(p)$ is a random closed set as introduced in [6].

Space $\mathrm{K}$ of all compact subsets of $X$ can be metrized by the Hausdorff distance:

$$
\rho_{\mathrm{H}}\left(K, K_{1}\right)=\inf \left\{r>0: K \subset K_{1}^{r}, K_{1} \subset K^{r}\right\}
$$

where $K, K_{1} \in \mathcal{K}$

$$
K^{r}=\{x: b(x, r) \cap K \neq \emptyset\}
$$

is the $r$-parallel set to $K$ and $b(x, r)$ is the ball of radius $r$ centered at $x$. For each set $M \subset X$ we shall write $\operatorname{cl}(M)$, Int $M$ and $\partial M$ for its closure, interior and boundary respectively.

\section{Strong CONSISTENCY}

The estimator $H_{n}(p)$ is said to be strongly consistent if

$$
\rho_{\mathrm{H}}\left(H_{n}(p) \cap K_{0}, H(p) \cap K_{0}\right) \rightarrow 0 \quad \text { a.s. as } \quad n \rightarrow \infty
$$

for each compact $K_{0}$. The distance $\rho_{\mathrm{H}}\left(H_{n}(p) \cap K_{0}, H(p) \cap K_{0}\right)$ is a random variable, since $H_{n}(p) \cap K_{0}$ is a random closed set, and the function $\rho_{\mathrm{H}}(\cdot, K)$ is continuous.

Theorem 2.1. Suppose that, for each compact $K_{0}$,

$$
\eta_{n}=\sup _{x \in K_{0}}\left|h_{n}(x)-h(x)\right| \rightarrow 0 \quad \text { a.s. as } \quad n \rightarrow \infty .
$$

The estimator $H_{n}(p)$ is strongly consistent if

$$
H(p) \subset \operatorname{cl}(H(p-))
$$

where $H(p-)=\{x: h(x)<p\}$. If for each $x$ there exists a sequence $n(k)$ such that $h_{n(k)}(x)>h(x)$ a.s., then (2.2) is also a necessary condition. Moreover, if (2.2) is valid for each $p \in\left[c_{1}, c_{2}\right]$, then

$$
\sup _{c_{1} \leq p \leq c_{2}} \rho_{\mathrm{H}}\left(H_{n}(p) \cap K_{0}, H(p) \cap K_{0}\right) \rightarrow 0 \quad \text { a.s. as } \quad n \rightarrow \infty \text {. }
$$


Proof. To simplify notations suppose that $X$ is compact, and $K_{0}=X$.

Sufficiency. It is evident that the function

$$
\phi(\varepsilon)=\rho_{\mathrm{H}}(H(p+\varepsilon), H(p)) .
$$

is right-continuous, non-increasing for $\varepsilon<0$ and non-decreasing for $\varepsilon>0$. Note that $\phi(0)=0$. Moreover, $(2.2)$ yields $\rho_{\mathrm{H}}(H(p), H(p-))=0$, that is $\phi$ is continuous at zero.

Evidently, $H_{n}(p) \subset\left\{x: h(x)<p+\eta_{n}\right\}$. Similarly,

$$
\begin{aligned}
H(p) \subset H\left(p-\eta_{n}\right)^{\phi\left(\eta_{n}\right)} & =\cup\left\{b\left(x, \phi\left(\eta_{n}\right)\right): h(x)<p-\eta_{n}\right\} \\
& \subset H_{n}(p)^{\phi\left(\eta_{n}\right)} .
\end{aligned}
$$

Hence, (2.1) yields

$$
\rho_{\mathrm{H}}\left(H_{n}(p), H_{n}\right) \leq \phi\left(\eta_{n}\right) \rightarrow 0 \quad \text { a.s. as } \quad n \rightarrow \infty .
$$

Furthermore, (2.3) follows from the monotonicity of $H$ and its estimator.

Necessity. Let $H(p) \not \subset \operatorname{cl}(H(p-))$. Then there exists a point $x$, such that $h(x)=p$ and $\rho\left(x, \operatorname{cl}(H(p-))=\delta>0\right.$. Then $h_{n(k)}(x)>h(x)=p$ by the condition of Theorem. Therefore, $x \notin H_{n(k)}(p)$, whence $\rho\left(H_{n(k)}(p), H(p)\right)>\delta$, contrary to the consistency of the estimator $H_{n}(p)$.

Heuristic, (2.2) for all $p$ means that the function $h$ is not constant on any open subset of $X$.

\section{Limit TheOREMS}

Let us proceed to find the asymptotic distribution of the Hausdorff distance between $H_{n}(p)$ and $H(p)$. First, for any function $f: X \mapsto \mathbf{R}$ and a compact set $K_{0}$ introduce the functional

$$
\Phi(f)=\rho_{\mathrm{H}}\left(H(p ; f) \cap K_{0}, H(p) \cap K_{0}\right)
$$

where

$$
H(p ; f)=\{x: h(x) \leq p+f(x)\} .
$$

Evidently, $\rho_{\mathrm{H}}\left(H_{n}(p), H(p)\right)=\Phi\left(\zeta_{n}(\cdot) / a_{n}\right)$. Let us put

$$
\begin{aligned}
K(p) & =\left\{x \in K_{0}: h(x)=p\right\} \\
K(p ; \varepsilon) & =\left\{x \in K_{0}:|h(x)-p| \leq \varepsilon\right\}, \quad \varepsilon>0 .
\end{aligned}
$$

If $\sup _{x \in X}|f(x)|=\varepsilon$, then $H(p ; f)=H\left(p ;\left.f\right|_{\varepsilon}\right)$, where

$$
\left.f\right|_{\varepsilon}(x)=\left\{\begin{array}{ll}
f(x) & , \quad x \in K(p ; \varepsilon) \\
0 & , \text { otherwise }
\end{array} .\right.
$$

Therefore, in this case $\Phi(f)=\Phi\left(\left.f\right|_{\varepsilon}\right)$.

Following Borovkov [1] and Molchanov [7] a functional $\Phi$ (in general not necessarily defined by (3.1)) is said to be continuously differentiable if there exists a functional $\Phi^{\prime}$ 
such that for each continuous function $f$ and each sequence $\left\{f_{\delta}\right\}$, such that $f_{\delta}$ converges uniformly on $K_{0}$ to $f$ as $\delta \downarrow 0$

$$
\begin{aligned}
\delta^{-1} \Phi\left(\delta f_{\delta}\right) & \rightarrow \Phi^{\prime}(f) \quad \text { as } \quad \delta \downarrow 0 \\
\Phi^{\prime}\left(f_{\delta}\right) & \rightarrow \Phi^{\prime}(f) \quad \text { as } \quad \delta \downarrow 0 \\
\Phi^{\prime}\left(\left.f\right|_{\varepsilon}\right) & \rightarrow \Phi^{\prime}\left(\left.f\right|_{0}\right) \quad \text { as } \quad \varepsilon \downarrow 0
\end{aligned}
$$

Theorem 3.1. Let the random field (1.3) converge weakly to a continuous random field $\zeta$. Suppose that the functional (3.1) is continuously differentiable. Then $a_{n} \rho_{\mathrm{H}}\left(H_{n}(p), H(p)\right)$ converges in distribution to $\Phi^{\prime}\left(\left.\zeta\right|_{0}\right)$.

Proof. Evidently, $a_{n} \rho_{\mathrm{H}}\left(H_{n}(p), H(p)\right)=a_{n} \Phi\left(\left.\left(a_{n}^{-1} \zeta_{n}\right)\right|_{\eta_{n}}\right)$, where $\eta_{n}$ has been defined in (2.1). It is easy to show that the random variable $a_{n} \Phi\left(\left.\left(a_{n}^{-1} \zeta_{n}\right)\right|_{\delta}\right)$ converges in distribution to $\Phi^{\prime}\left(\left.\zeta\right|_{\delta}\right)$ for each sufficiently small $\delta$. Now the statement of Theorem follows from (3.2)$(3.4)$.

Let us now find a representation for the derivative $\Phi^{\prime}$ of the functional (3.1). For this, define

$$
\omega_{h}(x, t)=\inf \left\{h(y)-h(x): \rho(x, y) \leq t, y \in K_{0}\right\}, \quad x \in K_{0} .
$$

Theorem 3.2. Suppose that the following conditions are valid:

(i) For each $x, \omega_{h}(x, t)$ is continuous for $t$ belonging to a certain neighborhood of the origin;

(ii) Function $\omega_{h}(x, t)$ is differentiable at $t=0$ uniformly for $x \in K(p ; \varepsilon)$ and its derivative $L(x)=\omega_{h}^{\prime}(x, 0)$ is upper semicontinuous and non-vanishing on $K(p ; \varepsilon)$.

Then the functional $\Phi$ is continuously differentiable,

$$
\Phi^{\prime}(f)=\sup _{x \in K(p)}|f(x) / L(x)|
$$

and $a_{n} \rho_{\mathrm{H}}\left(H_{n}(p) \cap K_{0}, H(p) \cap K_{0}\right)$ converges in distribution to $\sup _{x \in K(p)}|\zeta(x) / L(x)|$.

Proof. Let us verify the differentiability of $\Phi$ and find its derivative. Let $M_{+}(\delta)=$ $\left\{x \in K_{0}: f_{\delta}>0\right\}$ and $M_{-}(\delta)=\left\{x \in K_{0}: f_{\delta}<0\right\}$, where $f_{\delta}$ is the function from (3.2) and (3.3). Furthermore, put

$$
S(\delta)=\left\{x \in M_{+}(\delta): h(x) \in\left(p, p+\delta f_{\delta}(x)\right]\right\} \bigcup\left\{x \in M_{-}(\delta): h(x) \in\left(p+\delta f_{\delta}(x), p\right]\right\} .
$$

Then

$$
\begin{aligned}
\Phi\left(\delta f_{\delta}\right) & =\rho_{\mathrm{H}}\left(H\left(p ; \delta f_{\delta}\right) \cap K_{0}, H(p) \cap K_{0}\right) \\
& =\max \left(\sup _{x \in M_{+}(\delta) \cap S(\delta)} \rho\left(x, H(p) \cap K_{0}\right), \sup _{x \in M_{-}(\delta) \cap S(\delta)} \rho\left(x, H\left(p ; \delta f_{\delta}\right) \cap K_{0}\right)\right) .
\end{aligned}
$$

Put for any $\gamma<0$

$$
\bar{\omega}_{h}(x, \gamma)=\inf \left\{t \geq 0: \omega_{h}(x, t)=\gamma\right\}
$$


If $x \in M_{+}(\delta) \cap S(\delta)$, then $p=h(x)-\delta f_{\delta}(x) r_{\delta}(x)$, where $0 \leq r_{\delta} \leq 1$. Furthermore, $r_{\delta}(x)=0$ for $x \in K(p)$.

Then, for $x \in M_{+}(\delta) \cap S(\delta)$,

$$
\begin{aligned}
\rho(x, H(p)) & =\inf \left\{t \geq 0: x \in\left(H(p) \cap K_{0}\right)^{t}\right\} \\
& =\inf \left\{t \geq 0: x \in b(y, t), h(y) \leq p, y \in K_{0}\right\} \\
& =\inf \left\{\delta \geq 0: \omega_{h}(x, \delta) \leq-\delta f_{\delta}(x) r_{\delta}(x)\right\} \\
& =\bar{\omega}_{h}\left(x,-\delta f_{\delta}(x) r_{\delta}(x)\right) .
\end{aligned}
$$

Similarly, for each $x \in M_{-}(\delta) \cap S(\delta)$,

$$
\rho\left(x, H\left(p ; \delta f_{\delta}\right) \cap K_{0}\right)=\bar{\omega}_{h-\delta f_{\delta}}\left(x, \delta f_{\delta}(x) r_{\delta}(x)\right) .
$$

Thus, $\Phi\left(\delta f_{\delta}\right)=\max \left(\phi_{+}(\delta), \phi_{-}(\delta)\right)$, where

$$
\begin{aligned}
& \phi_{+}(\delta)=\sup _{x \in M_{+}(\delta) \cap S(\delta)} \bar{\omega}_{h}\left(x,-\delta f_{\delta}(x) r_{\delta}(x)\right), \\
& \phi_{-}(\delta)=\sup _{x \in M_{-}(\delta) \cap S(\delta)} \bar{\omega}_{h-\delta f_{\delta}}\left(x, \delta f_{\delta}(x) r_{\delta}(x)\right) .
\end{aligned}
$$

Let us show that the function $\phi_{+}(\delta)$ is differentiable at zero and find $\phi_{+}^{\prime}(0)$. It follows from (i) and (ii) that $\bar{\omega}_{h}(x, \gamma)$ is differentiable at $\gamma=0$ uniformly for $x \in K(p ; \varepsilon)$, and $\bar{\omega}_{h}^{\prime}(x, 0)=1 / L(x)$. Since the functions $f$ and $r_{\delta}$ are bounded and $f_{\delta}$ converges to $f$ uniformly, we get

$$
\bar{\omega}_{h}\left(x,-\delta f_{\delta}(x) r_{\delta}(x)\right)=\bar{\omega}_{h}^{\prime}(x, 0)\left[-\delta f_{\delta}(x) r_{\delta}(x)\right]+\delta \kappa(x, \delta),
$$

where $\sup _{x \in K(p ; \varepsilon)} \kappa(x, \delta) \rightarrow 0$ as $\delta \rightarrow 0$. Therefore,

$$
\begin{aligned}
\phi_{+}^{\prime}(0) & =\lim _{\delta \downarrow 0} \sup _{x \in M_{+}(\delta) \cap S(\delta)} \bar{\omega}_{h}^{\prime}(x, 0)\left[-f_{\delta}(x) r_{\delta}(x)\right] \\
& =\lim _{\delta \downarrow 0} \sup _{x \in M_{+}(\delta) \cap S(\delta)}|f(x) / L(x)|
\end{aligned}
$$

Note that $\left\{x: f(x)>\alpha_{\delta}\right\} \subset M_{+}(\delta) \subset\left\{x: f(x)>-\alpha_{\delta}\right\}$, where

$$
\alpha_{\delta}=\sup _{x \in K_{0}}\left|f(x)-f_{\delta}(x)\right| \rightarrow 0 \text {. }
$$

The continuity of $f$ and (ii) yield the upper semicontinuity of the function $|f(x) / L(x)|$. Hence

$$
\phi_{+}^{\prime}(0)=\sup _{x \in K(p), f(x) \geq 0}|f(x) / L(x)| .
$$

Let us proceed to find the derivative $\phi_{-}^{\prime}(0)$. Clearly,

$$
\delta^{-1}\left|\omega_{h-\delta f_{\delta}}(x, t)-\omega_{h}(x, t)\right| \leq \sup \left\{\left|f_{\delta}(x)-f_{\delta}(y)\right|: \rho(x, y) \leq t\right\} .
$$

Thus,

$$
\left|\omega_{h-\delta f_{\delta}}(x, t)-\omega_{h}^{\prime}(x, 0) t\right| \leq h \Delta(x, \delta)+o(\delta)+o(t) \quad \text { as } \quad t \rightarrow 0
$$


where $\Delta(x, \delta) \rightarrow 0$ as $\delta \rightarrow 0$ uniformly for $x \in X$. For $\gamma=\delta f_{\delta}(x) r_{\delta}(x)$ we get

$$
\begin{aligned}
\delta^{-1} \bar{\omega}_{h-\delta f_{\delta}}(x, \gamma) & =\delta^{-1} \inf \left\{t \geq 0: \omega_{h-\delta f_{\delta}}(x, t)=\gamma\right\} \\
& =\inf \left\{t \geq 0: \omega_{h-\delta f_{\delta}}(x, t \delta)=\gamma\right\} \\
& \leq \inf \left\{t \geq 0: \omega_{h}^{\prime}(x, 0) t \delta=\gamma+\delta \Delta(x, \delta)+o(\delta)+o(t \delta)\right\} \\
& \leq \inf \left\{t \geq 0: \omega_{h}^{\prime}(x, 0) t=f_{\delta}(x) r_{\delta}(x)+c(\delta)\right\}
\end{aligned}
$$

where $c(\delta) \rightarrow 0$ as $\delta \rightarrow 0$.

A similar bound from below is also true. From (ii) we get

$$
\begin{aligned}
\phi_{-}^{\prime}(0) & =\lim _{\delta \downarrow 0} \sup _{x \in M_{-}(\delta) \cap S(\delta)} f_{\delta}(x) r_{\delta}(x) / L(x) \\
& =\sup _{x \in K(p), f(x) \leq 0}|f(x) / L(x)|
\end{aligned}
$$

From this and (3.7) it follows that the derivative $\Phi^{\prime}(f)$ is determined by (3.6). The functional $\Phi^{\prime}$ satisfies the conditions (3.3) and (3.4). Now the limit theorem for the Hausdorff distance follows directly from Theorem 3.1 .

If $X=\mathbf{R}^{d}$ and $h$ is continuously differentiable, then it is possible to find the corresponding derivative $L(x)$.

Theorem 3.3. Suppose that $K_{0}=\operatorname{cl}\left(\right.$ Int $\left.K_{0}\right)\left(K_{0}\right.$ is regular closed) and $K_{0}$ has $\mathcal{C}^{1}$ boundary $\partial K_{0}$. Let $\mathbf{n}(x)$ be the unit outer normal vector to $K_{0}$ at $x \in \partial K_{0}$. Furthermore, let $h$ be continuously differentiable at a neighborhood of $K(p)$. Put

$$
\mathbf{v}(x)=-\operatorname{grad} h(x)=-\left(\frac{\partial h}{\partial x_{1}}, \ldots, \frac{\partial h}{\partial x_{d}}\right) .
$$

Then the conditions (i) and (ii) are valid and

$$
L(x)=\left\{\begin{array}{ll}
\|\mathbf{v}(x)\| & , \quad x \in \operatorname{Int} K_{0} \\
\|\mathbf{v}(x)\| & , \quad x \in \partial K_{0}, \phi(x) \geq \frac{\pi}{2} \\
\|\mathbf{v}(x)\| \sin \phi(x) & , \quad x \in \partial K_{0}, \phi(x)<\frac{\pi}{2}
\end{array} .\right.
$$

where $\phi(x)$ is the angle between $\mathbf{v}(x)$ and $\mathbf{n}(x)$.

Proof follows from the Taylor expansion of $h(y)-h(x)$ in (3.5).

In the analogous way also a system of inequalities $\left\{x: h_{1}(x) \leq p_{1}, \ldots, h_{m}(x) \leq p_{m}\right\}$ with $p_{i}>0$ can be considered. This case can be reduced to the case (1.1) for the function $h(x)=\max _{1 \leq i \leq m} h_{i}(x) / p_{i}$.

It is possible also to consider analogs of the Hausdorff distance by

$$
\rho_{\mathrm{H}}^{B}\left(K, K_{1}\right)=\inf \left\{r>0: K \subset K_{1} \oplus r B, K_{1} \subset K \oplus r B\right\}
$$

where $\oplus$ is the Minkowski addition and $B$ is a convex set containing the origin as its interior point. In the usual definition of the Hausdorff distance $B$ is the unit ball. Then Theorem 3.2 is valid after replacing in $(3.5) \rho(x, y) \leq \delta$ by $y \in x+\delta B$. 


\section{EXAMPLES}

In the simplest case $h$ is a monotone (say increasing) function on the line. Then the estimator $H_{n}(p)$ is strongly consistent if $h$ is continuous at the point $x_{p}=\sup \{x: h(x) \leq p\}$. Furthermore, $a_{n} \rho_{\mathrm{H}}\left(H_{n}(p), H(p)\right)$ converges weakly to $\left|\zeta\left(x_{p}\right) / L\left(x_{p}\right)\right|$. If $h(x)=x$, then $L(x)=-1$ for $x \in(0,1)$. Thus, for $p \in(0,1)$, the limit is distributed as $|\zeta(p)|$. If $h(x)=x^{2}$, then $L(x)=-2|x|$, and $a_{n} \rho_{\mathrm{H}}\left(H_{n}(p), H(p)\right)$ converges in distribution to $\max (|\zeta(\sqrt{p})|,|\zeta(-\sqrt{p})|) / 2 \sqrt{p}$.

Furthermore, in $\mathbf{R}^{d}$ put $h(x)=\|x\|^{\alpha}, \alpha>0$. Then $L(x)=-\alpha\|x\|^{\alpha-1}$ inside Int $K_{0}$, and the weak limit of $a_{n} \rho_{\mathrm{H}}\left(H_{n}(p), H(p)\right)$ is equal to $\sup _{\|x\|=p}|\zeta(x)| /\left(\alpha p^{\alpha-1}\right)$.

Let us consider also another example related to the theory of random closed sets. Let $\xi(x)$ be the support function of a random compact set $A$, that is

$$
\xi(x)=\sup \{(u \cdot x): u \in A\}
$$

where $(u \cdot x)$ denotes the scalar product. We suppose that $\|A\|=\sup \{\|x\|: x \in A\}$ has a finite expectation. Then $h(x)=\mathbf{E} \xi(x)$ is the support function of the Aumann expectation (mean body) $\mathbf{E} A$ of $A$, see $[12,11]$. For $p=1$, the corresponding set $H(1)$ defined by (1.1) is a so-called polar set $(\mathbf{E} A)^{\circ}$ of $\mathbf{E} A$, see [10]. Suppose that $\mathbf{E} A$ contains the origin as an interior point.

Let $\xi_{1}, \ldots, \xi_{n}$ be the support functions of iid copies of $A$. Then the set

$$
H_{n}(p)=\left\{x: h_{n}(x)=\frac{1}{n} \sum \xi_{i}(x) \leq p\right\}=p\left(\frac{1}{n}\left(A_{1} \oplus \cdots \oplus A_{n}\right)\right)^{\circ}
$$

is a strongly consistent estimator of $H(p)=p(\mathbf{E} A)^{\circ}$. Note that $h_{n}$ is the support function of $\left(A_{1} \oplus \cdots \oplus A_{n}\right) / n$.

Pick compact $K_{0}$ such that $(\mathbf{E} A)^{\circ} \subset$ Int $K_{0}$. If the boundary of $\mathbf{E} A$ is smooth $\left(\mathcal{C}^{1}\right)$, then the function (3.5) satisfies the conditions of Theorem 3.2 with $L(x)=\|x\|^{-1} h(x)$. It follows from Theorem 3.2 and the central limit theorem for Minkowski sums of random sets [12] that

$$
\left.\sqrt{n} \rho_{\mathrm{H}}\left(H_{n}(1) \cap K_{0}, H(1)\right)=\sqrt{n} \rho_{\mathrm{H}}\left(\left(A_{1} \oplus \cdots \oplus A_{n}\right) / n\right)^{\circ} \cap K_{0},(\mathbf{E} A)^{\circ}\right)
$$

converges weakly to

$$
\sup \left\{\zeta(x)\|x\|: x \in \partial(\mathbf{E} A)^{\circ}\right\}
$$

where $\zeta$ is the centered Gaussian random field on $\mathbf{R}^{d}$ with the same covariance as the support function $\xi$ of $A$.

A solution of inequality was used in [8] to estimate the shape of a deterministic grain in a Boolean model. For this, the function $h$ is determined through the covariance function of the Boolean model. To avoid technicalities, we mention only that Theorem 3.2 can be applied to establish a limit theorem for the corresponding set-valued estimator. 


\section{REFERENCES}

[1] Borovkov, A.A. (1984) Statistique Mathematique. Mir, Trad. Francais, Moscow, 1987.

[2] EdDy, W.F. (1984) Set-valued orderings for bivariate data. In: Stochastic Geometry, Geometric Statistics, Stereology (Eds. R.Ambartzumian, W.Weil) Leipzig, Teubner, 79-90 (Teubner Texte zur Mathematik, B.65).

[3] Hartigan, J.A. (1975) Clustering Algorithms. Wiley, New York.

[4] Hartigan, J.A. (1987) Estimation of a convex density contour in two dimensions. J. Amer. Stat. Assoc. 82, 267-270.

[5] Korostelev, A.P. And Tsybakov, A.B. (1993) Minimax Theory of Image Restoration. Springer, New York.

[6] Matheron, G. (1975) Random Sets and Integral Geometry. Wiley, New-York.

[7] Molchanov, I.S. (1990) Empirical estimation of distribution quantiles of random closed sets Theory Probab. Appl. 35, 594-600.

[8] Molchanov, I.S. (1992) Handling with spatial censored observations in statistics of Boolean models of random sets. Biom. J. 34, 617-631.

[9] Nolan, D. (1991) The excess-mass ellipsoid. J. Multiv. Anal. 39, 348-371.

[10] Rockafellar, R.T. (1970) Convex Analysis. Princeton University Press, Princeton, NJ.

[11] Vitale, R. (1988) On alternate formulation of mean value for random geometric figures. J. Microscopy 151, 197-204.

[12] Weil, W. (1982) An application of the central limit theorem for Banach-spacevalued random variables to the theory of random sets. Z. Wahrscheinlichkeitstheorie 60, 203-208. 